- Ospedale Civico Lugano

(Dr. F. Bornatico-Valsangiacomo, Dr. M. Pons)

- Schmerzklinik Kirschgarten, Basel

(PD Dr. D. Schneider-Helmert)

- Universitätsspital (Inselspital) Bern

(PD Dr. C. Bassetti, Prof. M. Gugger)

- Universitätsspital Zürich (PD Dr. K. Bloch)

Änderungen in der personellen Zusammensetzung des Teams der Schlafzentren sollten der SGSSC laufend gemeldet werden. Alle 5 Jahre muss die Leitung eines Schlafzentrums für Schlafmedizin bestätigen, dass sich keine wesentlichen Änderungen ergeben haben. Bei wesentlichen betrieblichen oder personellen Änderungen oder sonstigen Problemen wird die Zertifizierung, evtl. im Rahmen einer Laborvisite, überprüft.

\section{Neue Anträge}

Neue Akkreditierungsanträge können an den Präsidenten/die Präsidentin der SGSSC gerichtet werden. Die Evaluation der neuen Anträge ist (wie bis jetzt) kostenpflichtig und wird in halbjährlichen Abständen (das nächste Mal im Sommer 2000) erfolgen. In einem ersten Schritt wird die Zertifizierung mittels eines Fragebogens durchgeführt, welcher von der Webpage der SGSSC heruntergeladen werden kann. Der Antragsteller bestätigt mit seiner Unterschrift, die Fragen wahrheitsgetreu beantwortet zu haben. Die SGSSC kann ohne Angabe von Gründen eine Laborvisite anordnen, wenn dies als nötig erachtet wird.

Die Liste der akkreditierten Zentren wird laufend in der Webpage der SGSSC (www.swiss-sleep.ch) aktualisiert und kann im Sekretariat der SGSSC verlangt werden.

\section{Literatur}

1 Gugger M. Einleitende Bemerkungen zu den «Richtlinien zur Zertifizierung von Zentren für Schlafmedizin zur Durchführung von Polysomnographien». Schweiz Ärztezeitung 1998;79:2603.

2 Gugger M, Bassetti C, Bloch K, Blois R, Colomb E, WirzJustice A, Zagury S. Richtlinien zur Zertifizierung von "Zentren für Schlafmedizin» zur Durchführung von Polysomnographien. Schweiz Ärztezeitung 1998;79:2605-9.

\section{Centres de médecine du sommeil accrédités en Suisse}

\section{Bassetti}

ral de l'Intérieur le mandat d'élaborer, en collaboration avec les Sociétés Suisses de "Neurologie», de "Neurophysiologie clinique», de "Pneumologie» et de "Psychiatrie et Psychothérapie», des critères de certification des centres de médecine du sommeil habilités à pratiquer les polysomnographies. Dans ce but, la SSRSMSC a mis sur pied un comité d'accréditation formé de représentants de ces sociétés actives dans le domaine de la médecine du sommeil. En septembre 1998 ont été publiées dans le Bulletin des médecins suisses $[1,2]$ les «Directives pour la certification de centres de médecine du sommeil».

\section{La procédure d'accréditation} (PSG) est une prestation obligatoire de l'assurance des prestations pour les soins médicaux, à condition que l'indication et l'exécution de la PSG se fassent dans des centres qualifiés. La Société Suisse de Recherche sur le Sommeil, de Médecine du Sommeil et de Chronobiologie (SSRSMSC) a reçu du Département Fédé-

Correspondance:

PD Dr Claudio Bassetti

Président du comité d'accréditation

de la Société suisse de recherche sur le sommeil

de Médecine du sommeil et de Chronobiologie (SSRSMSC)

Hôpital de l'Ile

CH-3010 Berne
La procédure d'accréditation a commencé au printemps 1999 avec la création, par la SSRSMSC, d'une nouvelle commission d'accréditation et avec l'élaboration d'un formulaire standardisé de demandes d'accréditation des différents centres. La commission d'accréditation se compose de trois représentants de chacune des sociétés actives dans le domaine de la médecine du sommeil, à savoir la neurologie, la pneumologie et la psychiatrie (PD Dr C. Bassetti, PD Dr K. Bloch, Dr R. Blois, Dr E. Colomb, Prof. P. A. Despland, Dr A. Knoblauch, PD Dr Savoy, PD Dr D. SchneiderHelmert, Dr S. Zagury). Aussi bien les trois régions linguistiques du pays que les institutions universitaires et privées ont en outre été prises en considération. 


\section{Les conditions requises}

Comme principales conditions requises pour la certification d'un laboratoire du sommeil - qui par la suite peut porter le nom de "Centre de médecine du sommeil» - ont été retenus les critères suivants, conformément aux directives pour la certification:

1. La direction est assurée par un médecin ayant suivi au minimum une formation de deux ans dans un centre de médecine du sommeil reconnu en Suisse ou à l'étranger et qui est porteur d'un titre FMH en neurologie, pneumologie ou psychiatrie.

2. Le nom de "Centre de médecine du sommeil" implique la possibilité de diagnostic et de traitement de l'ensemble des troubles du sommeil et de la vigilance (insomnies, hypersomnies, parasomnies, affections chronobiologiques). Ceci présuppose, entre autres, l'existence d'un service ambulatoire de consultations du sommeil et d'une équipe interdisciplinaire composée de spécialistes FMH en neurologie, pneumologie et psychiatrie, dont l'un des membres devrait être porteur d'un certificat EEG.

3. Pour des raisons de qualité, 60 PSG au moins doivent être effectuées chaque année.

4. Les PSG sont pratiquées par des techniciens/techniciennes au bénéfice d'une formation spécifique. Les enregistrements polysomnographiques ont lieu dans des locaux spécialement aménagés dans ce but. Le technicien/la technicienne doit surveiller en continu, par vidéo, la totalité de l'enregistrement.

Dans l'appréciation des laboratoires du sommeil déjà en place, qui effectuent des enregistrements polysomnographiques depuis des années, on s'est toujours efforcé de tenir compte de manière appropriée le principe du droit acquis (dispositions transitoires jusqu'au 1.1.2000), pour autant que les critères de qualité soient respectés. Dans quelques cas, l'accréditation n'a été accordée que "sous réserve» ou pour une période limitée dans le temps. Jusqu'à ce jour, aucun recours contre les décisions prises n'est parvenu à la SSRSMSC.

\section{Les centres de médecine du sommeil}

Nous dressons ci-dessous la liste des centres de médecine du sommeil accrédités et de leurs directeurs (Etat au 1.3.2000):

- Ärztehaus Fluntern

(Dr H. Ben Shmuel, Dr M. Gross)

- Hôpitaux universitaires, Bâle (Pr M. Solèr, Pr E. Holsboer-Trachsler)
- Centre de Médecine du Sommeil, Genolier (Dr J. Y. Sovilla, Dr P. Kehrer)

- Centre Lausannois de Médecine du Sommeil (Dr S. Zagury, Dr E. Colomb)

- Hôpital cantonal, Saint-Gall (Dr A. Knoblauch)

- Clinique Barmelweid (Dr H. Keller)

- Clinique Zurzach (PD Dr J. Schwander)

- Hôpital Lindenhof, Berne (Dr W. Bauer)

- Ospedale Civico, Lugano (Dr F. Bornatico-Valsangiacomo, Dr M. Pons)

- Clinique de la douleur Kirschgarten (PD Dr. D. Schneider-Helmert)

- Hôpital universitaire (Hôpital de l'Ile), Berne (PD Dr C. Bassetti, Pr M. Gugger)

- Hôpital universitaire, Zurich (PD Dr K. Bloch)

La SSRSMSC sera tenue au courant de toute modification de la composition du personnel des équipes des centres du sommeil. Tous les 5 ans, la direction du centre de médecine du sommeil doit confirmer à la SSRSMSC qu'aucun changement important n'est intervenu. En cas de changements importants en personnel ou dans la marche du service ou en cas de problèmes, la certification sera reconsidérée, éventuellement dans le cadre d'une visite du laboratoire.

\section{Nouvelles demandes d'accréditation}

Les nouvelles demandes d'accréditation peuvent être adressées au président/à la présidente de la SSRSMSC. L'évaluation des nouvelles demandes est (comme par le passé) soumise au paiement d'un émolument et se fera à 6 mois d'intervalle (prochaine évaluation en été 2000). Dans un premier temps la certification sera effectuée au moyen d'un questionnaire qu'on peut obtenir sur la Webpage de la SSRSMSC. Le requérant garantit par sa signature avoir répondu aux questions en toute sincérité. La SSRSMSC peut ordonner, sans avoir à en donner les motifs, une visite de laboratoire, si elle la considère nécessaire.

La liste des centres accrédités est tenue à jour sur la Webpage de la SSRSMSC (www.swiss-sleep.ch) et peut être obtenue au secrétariat de la SSRSMSC.

\section{Références}

1 Gugger M. Remarques préliminaires sur les «Directives pour l'accréditation des centres de médecine du sommeil dans la réalisation des polysomnographies». Schweiz Ärztezeitung 1998;79:2604.

2 Gugger M, Bassetti C, Bloch K, Blois R, Colomb E, WirzJustice A, Zagury S. Directives pour la certification de "centres de médecine du sommeil" pour l'exécution d'enregistrements polysomnographiques. Schweiz Ärztezeitung 1998;79:2610-4. 